\title{
Visually induced activity in human frontal motor areas during simple visuomotor performance
}

\author{
Gregor Thut,, CA Claude-Alain Hauert, ${ }^{2}$ Olaf Blanke, Stéphanie Morand,' Margitta Seeck,' \\ Sara L Gonzalez,' Rolando Grave de Peralta,' Laurent Spinelli,' Asaid Khateb,' Theodor Landis' \\ and Christoph M. Michel'
}

Functional Brain Mapping Laboratory and Plurifaculty Program of Cognitive Neuroscience, ' Department of Neurology,
University Hospital of Geneva, 24 Rue Micheli du Crest, CH-12II Geneva 14; ${ }^{2}$ Faculty of Psychology, University of Geneva, I2II Geneva, Switzerland

${ }^{\mathrm{CA}}$ Corresponding Author

Received 17 May 2000; accepted 22 June 2000

\begin{abstract}
Visuomotor tasks elicit neuronal activity in primate motor areas at relatively short latencies. Although this early activity embodies features of visual responses (short latency, stimulusdependency), its sensory nature has been questioned. We investigated neural correlates of visuomotor performance in human motor areas using scalp and intracranial event-related potential measures. A simple visuomanual reaction-time task evoked early potentials at $133-145 \mathrm{~ms}$ post-stimulus which occurred much earlier than the motor potentials of the same
\end{abstract}

region. The amplitude of the early potentials covaried with stimulus location and was independent of parameters of the motor response. Because of their timing, stimulus-dependency and characteristics of our behavioral task, the early potentials are suggested to reflect neuronal responses of sensory nature rather than processing related to pure motor aspects of the task. NeuroReport I I:2843-2848 (C) 2000 Lippincott Williams \& Wilkins.

Key words: Human electrophysiology; Intracranial recordings; Source localization; Surface EPs; Visuomotor transformation

\section{INTRODUCTION}

Various sensory stimuli and behavioral tasks elicit neuronal activity in higher-order cortical areas at latencies when primary sensory areas are still activated. Visual stimuli, for example, evoke early neuronal responses in monkey premotor or primary motor cortex when the visual stimulus instructs a movement [1] but also when no movement to the stimulus is required [2,3]. These responses in monkey occur as early as $65-170 \mathrm{~ms}$ (mean latencies) after visual stimulus onset [4] and generally precede movement-dependent activity of the same areas during visuomotor performance [5-8]. Moreover, many of the premotor and motor neurons with early activity respond selectively to particular attributes of the visual stimulus, e.g. stimulus location for stationary stimuli [5-8], stimulus direction for moving stimuli [3], or stimulus identity/complexity [2]. It is still debated whether these responses are of visual nature or whether they reflect the motor significance of the stimulus [1].

Only few human studies have addressed this issue. Two recent visuomotor experiments have demonstrated early premotor activity time-locked to the visual signal in the human brain using fMRI and TMS $[9,10]$. In the present study, we aimed to confirm later findings using event- related potential measures. We further investigated whether such short-latency activity covaries with stimulus features and wanted to provide more clues on its nature (visual vs motor).

\section{MATERIALS AND METHODS}

Twelve healthy right-handed subjects (six women, six men, 21-27 years of age) were recorded with multichannel scalp evoked potentials (EPs). In addition, three pharmaco-resistant epileptic patients participated in this study which had subdural electrodes implanted as part of their presurgical evaluations (YF: right-handed, male, 32 years of age; NB: right-handed, female, 41 years; AM: ambidextrous, female, 26 years). Electrical cortical stimulation via the subdural electrodes revealed normal cerebral organization with respect to language and motor functions in all patients (for details see $[11,12])$ and their epileptic foci were localized remote from the contacts investigated in the present study. MRI showed left parietal and right prefrontal tubers in YF, atrophy of the frontal part of the left insula in NB and atrophy of the left occipital lobe in AM. The neurological status of YF and NB was normal. AM had a right homonymous hemianopia (eccentricities $>20^{\circ}$ ), facial asymmetry, 
hypoesthesia of right leg and right-sided dysdiadococinesia. All participants gave their informed consent.

EEG was recorded with $1000 \mathrm{~Hz}$ from 48 equidistant scalp-electrodes in the healthy subjects and with $200 \mathrm{~Hz}$ from 64-100 subdural electrodes in the patients (left hemispheres only, bipolar montage), while they were performing unimanual index finger responses to lateralized visual stimuli. The design dissociated the spatial location of the stimulus from the side of the motor response and thus allowed to search for brain activity which covaries with stimulus features independently of manual response side (stimulus-dependent activity). The stimuli were black dots (visual angle $0.5^{\circ}$ ) which were delivered randomly either to the left or to the right of a central fixation cross (horizontal eccentricity $4^{\circ}$ ) on a gray computer screen. Stimuli appeared for $60 \mathrm{~ms}$ every 5-6s. The healthy subjects had to respond in one experimental block with the left and in the other block with the right index finger as fast as possible to either of the two stimuli. Response keys were placed on the left and the right of the body midline respectively. Only the right hand experimental block was tested in all patients. The healthy subjects performed 60 and the pa- tients 60-100 trials for each of the tested hemifield/hand pairings. Fixation was controlled through video monitoring and EOG recordings. Only trials in which fixation was maintained were included in the analysis.

Scalp EP analysis: Off-line processing of the scalp data consisted of several steps, fully described in earlier articles $[13,14]$. This resulted for each subject in artifact-free and average-referenced EPs, aligned to stimulus onset (individual stimulus-locked EPs). These data were subjected to two analysis steps: (1) a classical analysis of the individual scalp EP traces performed over frontal electrodes presumably covering areas with hand motor functions (Fig. 1a) and (2) a source localization analysis using a distributed linear inverse solution. Source analysis is needed because EP traces at given electrodes reflect the superposition of activity arising at different brain sites rather than focal activity under the recording site itself. Source localization procedures, instead, are intended to estimate the location of the generator(s) in the brain that have produced the surface potentials, and their behavior over time. Here, we applied a recently developed algorithm, called ELECTRA,
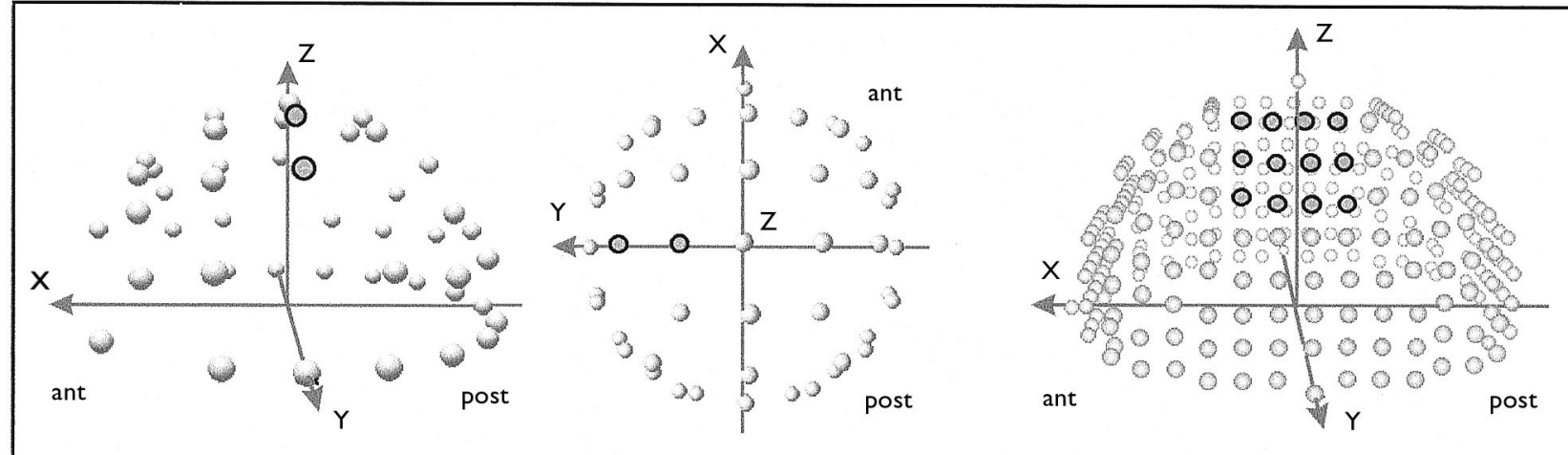

(c) Intracranial contacts in patients

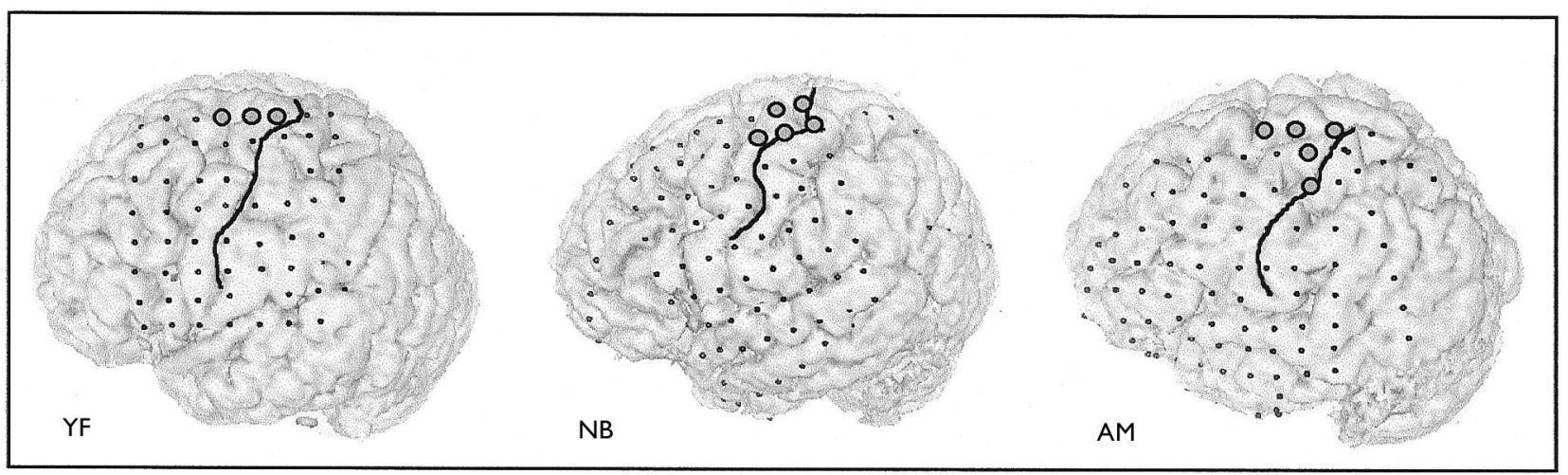

Fig. I. (a) 3D standard array of the 48 scalp electrodes. The frontal electrodes of interest, presumably localized over hand motor areas, are highlighted with dark gray circles. They correspond to electrodes $\mathrm{Cl}, \mathrm{C} 3$ of the international I0-10 system (ant: front of the head, post: back of the head). (b) Outer mantle of the 3D solution space used for calculation of the inverse solution based on the scalp recordings of the healthy subjects. Dark gray circles depict the frontal solution nodes of interest. (c) Subdural electrodes (small black points) rendered over the MRI of the patients. The position of the electrodes was determined using intraoperative photographs and 3D-MRI scans with the MRI-compatible electrodes in place. The black line indicates the central sulcus as determined by electrical cortical stimulation. Contacts of interest situated over areas with hand motor functions (where electrical cortical stimulation elicited movements of the contralateral fingers and wrist) are marked with dark gray circles. 
which restricts the set of possible solutions to the type of currents that can produce electrical measurements (EEG) $[13,15,16]$. That is, it attempts to estimate from the whole scalp voltage topography (EP map) a 3D potential distribution within the brain (intracranial potentials and not dipolar currents) which can thus directly be compared to recordings from intracranial electrodes. The advantages and limitations of ELECTRA have been explored by means of simulations [15]. In short, it has been shown that while the instantaneous ELECTRA solutions might be misleading, a waveshape analysis as the one used in this paper is quite reliable. This corresponds well with the known limitations of distributed inverse solutions $[15,16]$. ELECTRA was calculated for a solution space of 1152 nodes homogeneously distributed in the innermost compartment of a three shell spherical model. The ELECTRA solutions were analyzed in a left frontal region. This region of interest (ROI) included 12 solution points of the outer mantle of the $3 \mathrm{D}$ solution space and the 12 points just underneath, localized just in front of the central midline (Fig. 1b).

Intracranial EP analysis: The intracranial data were realigned to stimulus onset and averaged to individual stimulus-locked EPs of the patients after rejection of artifact- and spike-contaminated single-trial epochs. The regions of interest included a total of 13 left frontal contacts (Fig. 1c). They were selected because electrical cortical stimulation of these contacts led, in all three patients, to contralateral hand movements with index finger involvement. At more anterior contacts no responses (YF) or contraversive eye-movement were evoked (NB, AM), while stimulation at posterior/inferior contacts led to somatosensory responses in the contralateral hand or arm (YF, NB, AM). The 13 frontal contacts were situated on the precentral gyrus as determined by intraoperative photographs and 3D-MRI scans with the implanted electrodes in place.

\section{RESULTS}

Behavior: Mean reaction times (RTs) were $270 \mathrm{~ms}$ for the healthy subjects (range 218-329) and 407, 362 and $255 \mathrm{~ms}$ for $\mathrm{YF}, \mathrm{NB}$ and $\mathrm{AM}$ respectively. Crossed and uncrossed stimulus-response conditions were compared using $t$-tests (S-R incompatible vs S-R compatible trials). These revealed that RTs did not differ between S-R compatible and S-R incompatible trials of either hand, neither for the healthy subjects (left index responses: left- vs right-sided stimuli ( $270 \pm 42$ vs $272 \pm 36): \mathrm{t}=-0.56$, ns, right index responses: left- vs right-sided stimuli ( $271 \pm 36$ vs $268 \pm 31): \mathrm{t}=0.77$, ns) nor for the patients (right index: left- $v s$ right-sided stimuli: YF (416 \pm 118 vs $398 \pm 99)$ : $\mathrm{t}=0.86$, ns; $\mathrm{NB}$ ( $365 \pm$ 78 vs $360 \pm 69): \mathrm{t}=0.39$, ns; AM (242 \pm 49 vs $266 \pm 54)$ : $\mathrm{t}=-1.8, \mathrm{~ns})$.

Scalp potentials: To determine the latencies of the earliest components over electrodes $\mathrm{C} 1$ and $\mathrm{C} 3$, we first examined the condition expected to evoke the largest neuronal responses (right visual field stimuli, right hand respon$\mathrm{se}=\mathrm{RVF} / \mathrm{RH}$ ). The results showed that the earliest scalp responses started at around $100 \mathrm{~ms}$ post-stimulus, confirming the presence of early activity over human frontal cortex.

In order to check whether these responses covary with stimulus location independently of manual response side, we compared them between the different conditions (ANOVAs with visual field and responding hand as withinsubject factors). Statistics were performed time-frame by time-frame in the time window between perception and action. Figure 2a illustrates the scalp EPs in the four conditions for electrode $\mathrm{C} 3$ and the statistical results. Waveforms and results for $\mathrm{C} 1$ were almost identical but less significant. The earliest component occurring between 100 and $175 \mathrm{~ms}$ co-varied with stimulus location: it showed higher amplitudes for contralateral than ipsilateral visual stimulation (visual field effects at peak: $\mathrm{F}(1,11)=50.7, p<$ 0.00001; for further statistical results see Fig. 2a, box plot).

(a) Scalp potentials

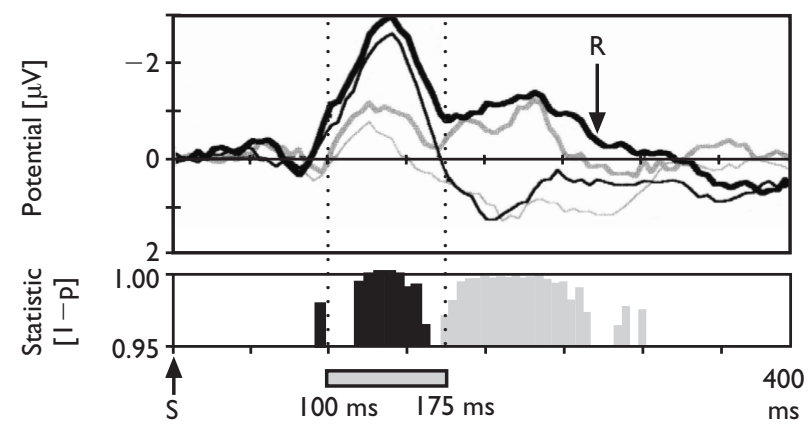

(b) Estimated potentials in depth

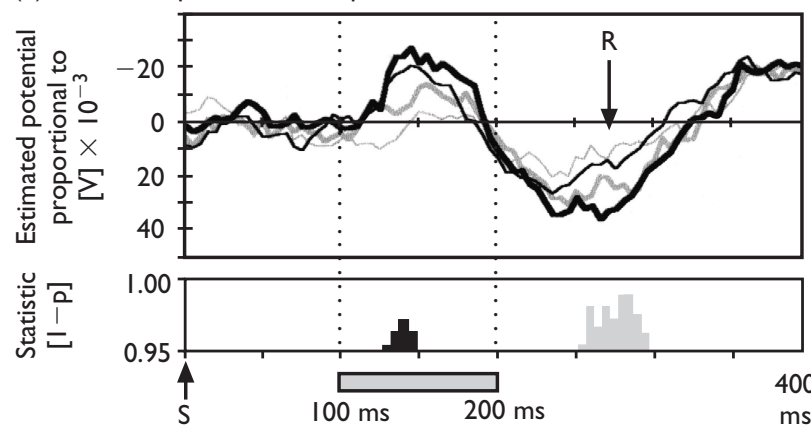

Potentials

Contralat. Vis. Field/Contralat. Hand

- Contralat. Vis. Field/lpsilat. Hand

Ipsilat. Vis. Field/Contralat. Hand

_ Ipsilat. Vis. Field/lpsilat. Hand

Statistics

Visual field effects Hand effects

Fig. 2. (a) Scalp potentials at electrode $\mathrm{C} 3$ for the four stimulus/ response conditions and (b) one type of estimated intracranial response pattern at the frontal solution nodes of interest (estimated using the ELECTRA algorithm from the healthy subjects' data). Cross-subject averages are shown in the time window between stimulus onset (marked by $S$ ) and index finger response (marked by R). The box plot below each line plot illustrates the corresponding statistical results (time frames of significant potential differences). Black areas depict time periods with visual field effects, gray areas intervals with hand effects (ANOVAs). Note that potentials covary at early time points with visual stimulus features (visual field of presentation) with stronger neuronal responses to contralateral than ipsilateral stimuli. At later time points, potentials covary with the responding hand. Negative potentials are up, positive down. 
At around $175 \mathrm{~ms}$, a second component appeared which differed in polarity between the two motor response conditions (amplitude differences: hand effects at peak: $\mathrm{F}(1,11)=19.5, p=0.001$, see also Fig. 2a).

Estimated intracranial potentials in healthy subjects: As for the EP trace analysis, we first analyzed the RVF/RH condition in order to determine the latencies of the earliest responses. Inspection of this condition revealed that a given solution point of the left frontal ROI rarely showed the same potentials over subjects, both regarding amplitude and latency of the different components. This is not surprising given the large interindividual variability in position and size of prerolandic motor areas [17]. Thus, in order not to mix solution points with different response patterns, we subjected the data to a k-means cluster-analysis and used a crossvalidation criterion to define the number of clusters that optimally explain the whole data set (for other EEG applications of this algorithm see $[13,14]$ ). The analysis revealed that all potentials of the RVF/RH condition are best explained by 12 clusters. The representative waveshapes of these clusters, which represent the 12 types of estimated potentials observed in the RVF/RH condition, all showed one or more components. The earliest components appeared around $75 \mathrm{~ms}$ post-stimulus.

Comparing each of these twelve waveshapes of the RVF/RH condition with the waveshapes of the same solution points and subjects of the other conditions (using ANOVAs) revealed that two of the 12 waveshapes covaried with stimulus location at early time points. The overall most significant waveshape type is shown in Fig. 2b. Similar to the surface traces, the early component showed higher amplitudes for contralateral than ipsilateral stimulus presentation (visual field effects at peak: $\mathrm{F}(1,8)=7.2, \quad p=0.028)$ and the late component higher amplitudes for contralateral than ipsilateral finger responses (hand effects at peak: $\mathrm{F}(1,8)=10.4, p=0.012$ ). However, in contrast to the surface traces on electrode C3, the late component had similar polarity for all conditions, suggesting that surface traces do not directly reflect focal activity in the region under the recording site. Note that this waveshape type was observed in nine of the 12 subjects (the cluster analysis grouped data of 9 subjects in the corresponding cluster), thus representing a finding which is characteristic for $75 \%$ of our healthy subject group. Its early stimulus-dependent components peaked at $145 \mathrm{~ms}$ post-stimulus.

Intracranial potentials in patients: At the frontal contacts of interest, task-related neuronal responses were observed in all patients (Fig. 3c). The early components showed peak latencies of $180 \mathrm{~ms}$ in YF, 120 and $170 \mathrm{~ms}$ in NB and 50 and $140 \mathrm{~ms}$ in AM (mean $133 \mathrm{~ms}$ ) and also co-varied with stimulus location. Statistics on the intracranial single-trial EEG data (unpaired $t$-tests) showed that amplitudes were significantly higher for contralateral than for ipsilateral hemifield presentation (effect at peak: YF: $\mathrm{t}=2.1, p=0.038$; NB: $\mathrm{t}=2.19, p=0.03, \mathrm{t}=2.25, p=0.026$; AM: $\mathrm{t}=2.15, p=$ $0.035, \mathrm{t}=2.16, p=0.034)$, fully compatible with the scalp and estimated intracranial results. This provides conclusive evidence that early potentials in human frontal areas are sensitive to visual parameters. Note that the intracranial potentials strongly resemble the estimated but less the scalp potentials, illustrating that the use of inverse solutions may reconstruct more precisely the temporal behavior of brain activity. This is demonstrated in the second component where differences between intracranial and scalp potentials are most obvious (Fig. $3 a-c)$.

\section{DISCUSSION}

Our results strongly suggest that there are early, stimulusdependent potentials in the human frontal cortex. The presence of movement-dependent activity recorded at the same sites at later time points in the healthy group suggests a location in an area with hand motor functions. Because no MR images are available in the healthy subjects and because of limitations in the model which we used for estimating the intracranial potentials (spherical head model instead of real head shape), however, a precise anatomical localization is not possible. The results of the patients confirm that the stimulus-dependent potentials are functionally localized in hand motor areas, given that electrical cortical stimulation at these sites induced contralateral finger movements. Anatomically, these sites are located over the precentral gyrus over either the premotor or the primary motor cortex as indicated by the patients' MR images.

The latencies of the electrical responses observed in the present study (range 50-180 ms, mean 133-145 ms) are in good agreement with single unit recordings demonstrating that visual stimuli can evoke neuronal activity in premotor or primary motor cortex at mean latencies of $65-170 \mathrm{~ms}$ [4] and with a recent TMS study in humans showing that single TMS pulses interfere with visuomotor task performance as early as $140-180 \mathrm{~ms}$ after visual cue onset when applied over the premotor cortex [9].

Our results furthermore mirror neurophysiological data in monkeys showing that there is early activity in both the premotor and primary motor cortex which is co-varying with stimulus features independently of the motor output [5-8]. However, it remained equivocal whether such stimulus-dependent activity in the monkey brain is truly sensory. This is because previous studies used variants of the pro-/antimovement paradigm to dissociate stimulusfrom movement-dependent activity. Pro-/antimovement paradigms, investigating movements towards a stimulus versus movements in an opposite direction, are confounded with the stimulus-response (S-R) compatibility effect, which is characterized by faster behavioral responses when stimulus and motor response match spatially (promovements) than when they do not match (antimovements). This effect most probably results from an early, automatic selection of the motor response towards the delivered stimulus, which is of use in promovement conditions but has to be discarded in antimovement conditions [18,19]. Accordingly, stimulus-dependent frontal activity in pro-/antimovement paradigms may also reflect such early, automatic motor processes of non-sensory nature $[5,8]$. In contrast to these previous studies, we applied a simple visuomotor reaction time task in which behavioral responses had to be given independently of stimulus side. Stimulus-dependent activity in such simple visuomotor tasks do more likely represent sensory responses than automatic motor activation processes for the 
(a) Scalp potentials

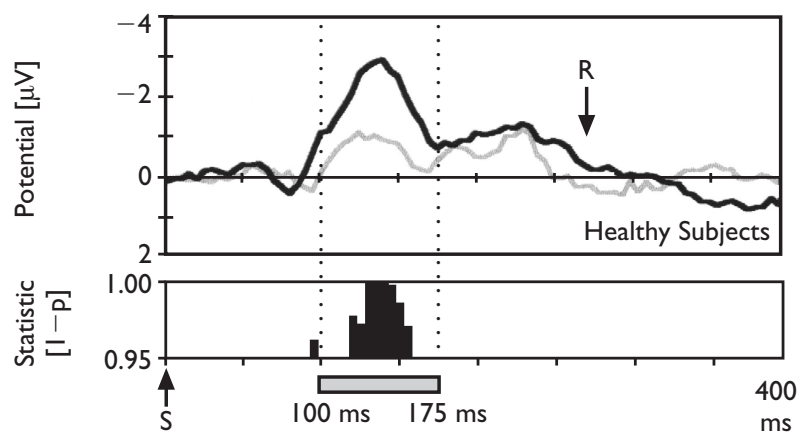

(b) Estimated potentials in depth
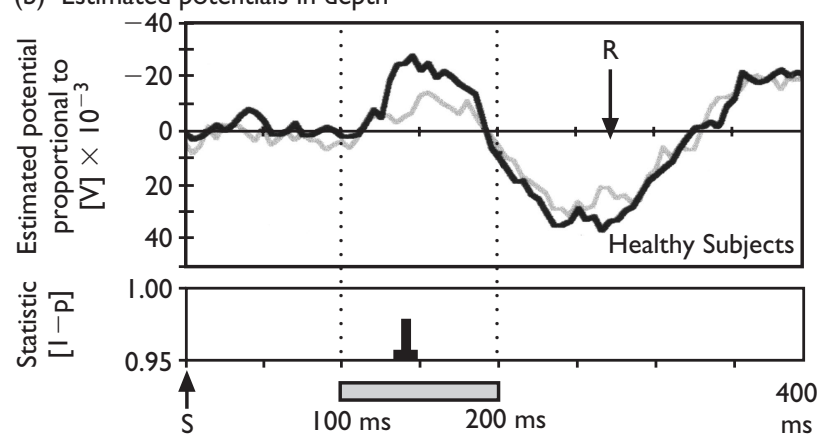

Potentials

Contralat. Vis. Field/Contralat. Hand

_ Ipsilat. Vis. Field/Contralat. Hand

Statistics

Visual field effects (c) Intracranial potentials
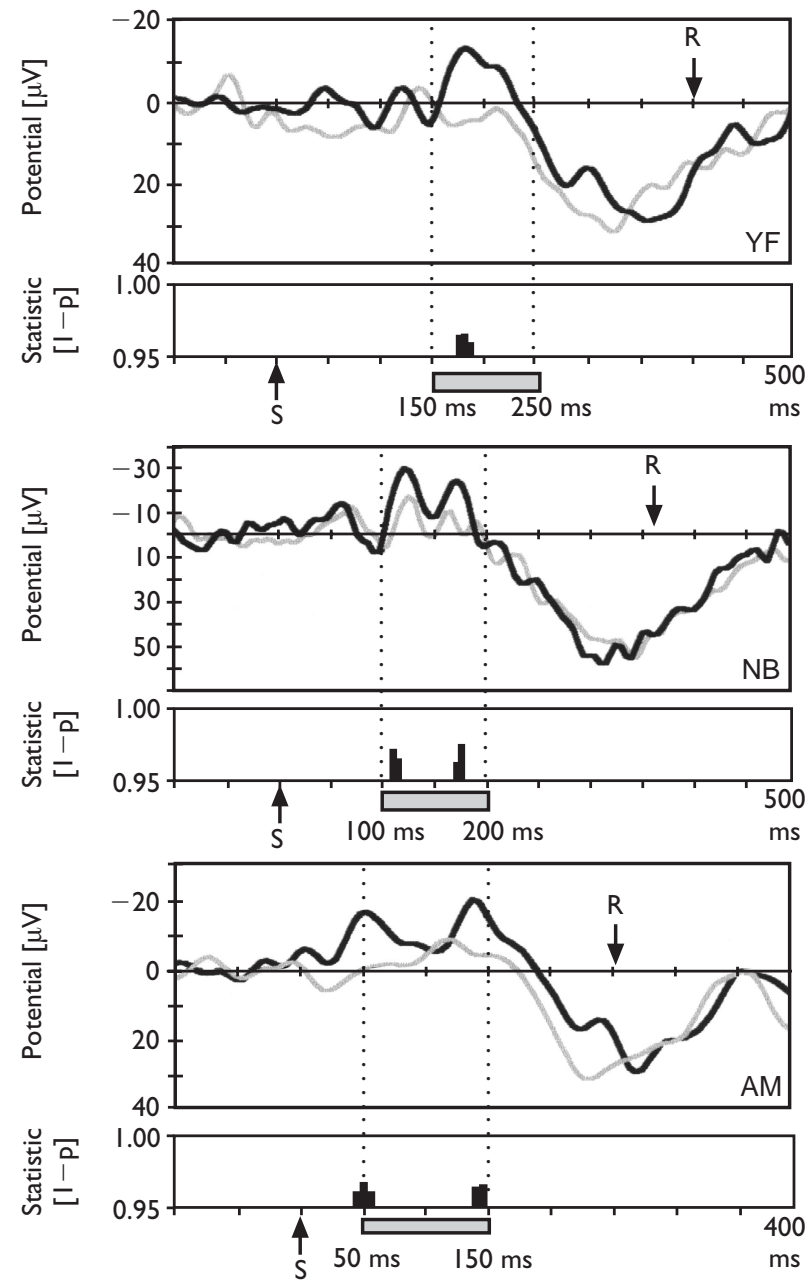

Fig. 3. Comparison of (a) scalp potentials and (b) estimated intracranial potentials at frontal sites (also shown in Fig. 2), with (c) the intracranial potentials at precentral motor contacts of patients YF, NB and AM. The two conditions also recorded in the patients are shown. S: Stimulus onset, R: time point of behavioral responses. Note the differences in scaling on the time axis due to differences in mean reaction times (healthy subjects: $270 \mathrm{~ms}$, YF: $407 \mathrm{~ms}$, NB: $362 \mathrm{~ms}$, AM: $255 \mathrm{~ms}$ ). The intracranial potentials most strongly resemble the ELECTRA response pattern, with early negative (delimited by dotted lines) and late positive components peaking shortly before the behavioral response. Note also the stimulus-dependency of the early components, statistically confirmed in both healthy subjects and patients using t-tests (box plots). Negative potentials are up, positive down.

following reasons. Since simple visuomotor tasks are not associated with behavioral S-R compatibility effects [20], an automatic translation of visual into spatially matching motor information is unlikely to be effective. Note also that there is no evidence for such behavioral effects in our study. Reaction times did not differ between S-R compatible and S-R incompatible trials of either hand, neither for the healthy group, nor for any of the patients. In addition, human EEG studies showed that neuronal correlates of automatic motor processes, reflected by incorrectly lateralized readiness potentials in pro-/antimovement paradigms, are largely abolished in simple response tasks [21]. Finally, these correlates appear at around 200-300 ms after stimulus onset $[18,21]$, much later than the early stimulusdependent activity observed in our subjects. There are thus more arguments for a sensory nature of our early stimulusdependent potentials, although further studies are needed to investigate what kind of sensory processing they represent, i.e., whether these potentials reflect true (higher order) visuospatial processing, the input of a simple, sensory trigger signal having a facilitatory function for subsequent visuomotor integration $[4,22]$, sensory-triggered attentional modulation [23], or other mechanisms.

\section{CONCLUSION}

Using event-related potential methods to study neuronal correlates of visuomotor performance, we confirmed the presence of early activity time-locked to the visual signal in human motor areas, as recently revealed by two experiments using fMRI and TMS [9,10]. In addition, we showed that the amplitude of these early neuronal responses covaried with visual stimulus attributes independently of parameters of the motor response. Based on its timing, stimulus-dependency and characteristics of our behavioral 
task, the early activity is suggested to reflect a neuronal response of sensory nature. This would be in line with the view that there are at least some neurons in primate motor areas that do play a role in sensory processing before the same areas are involved in processing related to pure motor aspects of the task [6,7].

\section{REFERENCES}

1. Boussaoud D, di Pellegrino G and Wise SP. Behav Brain Res 72, 1-15 (1996).

2. Murata A, Fadiga L, Fogassi L et al. J Neurophysiol 78, 2226-2230 (1997).

3. Wannier TMJ, Maier MA and Hepp-Reymond M-C. Neurosci Lett $\mathbf{9 8}$ 63-68 (1989).

4. Riehle A. Brain Res 540, 131-137 (1991)

5. Crammond DJ and Kalaska JF. J Neurophysiol 3, 1281-1284 (1994).

6. Shen L and Alexander GE. J Neurophysiol 77, 1171-1194 (1997).

7. Shen L and Alexander GE. J Neurophysiol 77, 1195-1212 (1997).

8. Zhang J, Riehle A, Requin J et al. J Neurosci 17, 2227-2246 (1997).
9. Schluter ND, Rushworth MFS, Mills KR et al. Neuropsychologia 37, 233-243 (1999).

10. Toni I, Schlutter ND, Joseph O et al. Cerebr Cortex 9, 35-49 (1999).

11. Blanke O, Morand S, Thut G et al. Neuroreport 10, 925-930 (1999).

12. Blanke O, Spinelli L, Thut G et al. Neuroreport, 11, 1907-1913 (2000).

13. Morand S, Thut G, Grave de Peralta R et al. Cerebr Cortex, 10, 817-825 (2000).

14. Thut G, Hauert C-A, Morand S et al. Exp Brain Res 128, 256-261 (1999).

15. Grave de Peralta R, Gonzalez SL, Morand S et al. Hum Brain Mapp 9, 1-12 (2000)

16. Michel CM, Grave de Peralta R, Lantz G et al. J Clin Neurophysiol 16, 239-266 (1999).

17. Roland PE and Zilles K. Trends Neurosci 17, 458-467 (1994).

18. Eimer M. J Exp Psychol HPP 21, 837-854 (1995).

19. Kornblum S, Hasbroucq T and Osman A. Psychol Rev 97, 253-270 (1990).

20. Anzola GP, Bertoloni G, Buchtel HA et al. Neuropsychologia 15, 295-302 (1977).

21. Wascher E and Wauschkuhn B. Electroencephalogr Clin Neurophysiol 99, 149-162 (1996).

22. Kwan HC, MacKay WA, Murphy JT et al. Brain Res 343, $24-35$ (1985).

23. Boussaoud D and Wise SP. Exp Brain Res 95, 15-27 (1993).

Acknowledgements: This study was supported by the Swiss National Science Foundation (grant no.II I4-45939.95) and the programme commun de recherche en génie biomédical 1999-2002. 